\title{
Posturographic Evaluation of Dizziness Complaining Patients under Suspicion to Develop Parkinson's Disease
}

\author{
Hyun Cho \\ Department of neurology, Daejeon Hankook Hospital, Daejeon, Korea. \\ E-mail: chohyun94@yahoo.co.kr
}

Received January $5^{\text {th }}$, 2011; revised January 20 $0^{\text {th }}$, 2011; accepted February $16^{\text {th }}, 2011$.

\begin{abstract}
Although the typical clinical signs of Parkinson disease $(P D)$ are tremor, rigidity, bradykinesia, and postural instability, $P D$ is preceded by a preclinical phase during which neuronal degeneration develops without typical symptoms. More general nonspecific symptoms including dizziness have also been described to predate the typical PD signs for several years. All subjects were selected among patients in the Willis Hospital (Pusan, South of Korea), with complaints of dizziness from September 2009 to September 2010 and the baseline neurological screening and clinical ENT examination, to which the results were within the normal range. At baseline, 113 participants underwent neurological screening and provided information on dizziness. Of those participants, 103 were enrolled including 63 subjects in the control group. We used posturography. It allows quantitative assessment of vestibular-spinal component of body balance. The parameter of average speed of pressure center displacement to the lateral plan (VMX) and antero-posterior plan (VMY), which presented statistically significant differences between the groups except VMX with closed eyes. $(p=0.008$ and $p=$ 0.012 , with closed eyes). With open eyes, only VMY showed significant difference between the groups $(p=0.010)$. In this study, the patients with dizziness and subjective complaints related to typical clinical signs of PD complaints presented higher instability in the orthostatic position than the control group of patients with dizziness and without such complaint. It could suggest that dizziness may be one symptom of preclinical PD and progress to overt postural instability. It is believed that a stepwise approach with a simple and inexpensive initial screening test of preclinical PD is required.
\end{abstract}

Keywords: Parkinson Disease, Postural Instability, Neuronal Degeneration, Posturography

\section{Introduction}

Although the typical clinical signs of Parkinson disease (PD) are tremor, rigidity, bradykinesia, and postural instability, PD is preceded by a preclinical phase during which neuronal degeneration develops without typical symptoms [1]. Evidence suggests that nonmotor abnormalities may occur during this phase, such as olfactory dysfunction, personality disturbances, and depression [26]. However, more general nonspecific symptoms including dizziness have also been described to predate the typical PD signs for several years [7]. Whitney [8] mentioned dizziness as one of the most frequent complaints in his study.

Dizziness is a subjective and nonspecific symptom with varied characteristics. It is caused by different pathophysiological mechanisms, and it may be a common complaint in different diseases.
The causes of dizziness are difficult to diagnose [9]. Currently, there is a wide range of complementary tests (radiological, audiometric, electrophysiological, posturographic, laboratory tests, etc), but none of them can properly assess the vestibular function. The most widely used tests in vestibular assessment are electronystagmographic tests (caloric tests, positional and rotation tests) and posturographic exams [10]. Posturography allows quantitative assessment of vestibular-spinal component of body balance. It is performed in static force platforms (stabilometry or statiokinesiometry) and dynamic forces (dynamic posturography). Stabilometry has been used for many authors in clinical practice and research studies [11].

We hypothesized that mild dopamine deficiency in preclinical PD might result in subtle subjective complaints including dizziness specifically related to motor 
function, and these complaints might be associated with an increased risk of PD in the future.

The present study intended to analyze the results of stabilometry of patients with dizziness and subjective complaints related to typical clinical signs of PD (tremor, rigidity, bradykinesia, and postural instability) and to compare them to the control group of patients with dizziness and without such complaints.

\section{Methods}

\subsection{Patients}

All subjects were selected among patients in the Willis Hospital (Pusan, South of Korea), with complaints of dizziness from September 2009 to September 2010 and the baseline neurological screening and clinical ENT examination, to which the results were within the normal range.

The following subjects were excluded from the study: those that presented clinical or past history of neurological disease including any parkinsonism or dementia, limiting or disabling musculoskeletal disease, otological or neurosurgical surgery, or if they had been using labyrinthic depressing drugs, benzodiazepine or anticonvulsant drugs.

At baseline, 113 participants underwent neurological screening and provided information on dizziness. Of the participants, 10 patients were excluded: six were diagnosed as PD according to UK Brain Bank criteria, two suffered from dementia and could not therefore be included in this study and two others had past history of otologic surgery. Finally, 103 were enrolled including 63 subjects in the control group.

\subsection{Assements of Subjective Complaints}

The participants who have self-reported complaints suggestive for parkinsonism were asked again by "yes" or "no" to indicate whether they ever experienced even one of 4 cardinal signs that are characteristic of PD: stiffness (rigidity), resting tremors of the head, arms, or legs (tremor), slowness of movement (bradykinesia), or postural instability [12].

Because absence of parkinsonism does not preclude the presence of 1 cardinal sign and to be maximally sure to evaluate subjects free of any parkinsonism, we only studied participants in whom none of the parkinsonian signs were found during a diagnostic workup comprising the Unified Parkinson Disease Rating Scale [13] and neurological examination.

\subsection{Assements of Dizziness}

We assessed dizziness in a stepwise fashion by asking participants to specify whether this concerned a near- fainting sensation (presyncope), spinning sensation (vertigo), feeling of imbalance (disequilibrium), or the perception of lacking control over leg movement.

We performed clinical ENT examination to exclude any specific ear, nose and/or throat affections. Patients were investigated aspects related to dizziness, tinnitus and hearing disorder. Vestibular assessment was carried out using electronystagmography.

\subsection{Posturography}

We used posturography. It allows quantitative assessment of vestibular-spinal component of body balance. It is performed in static force platforms (stabilometry or statiokinesiometry) and dynamic forces (dynamic posturography). Stabilometry has been used for many authors in clinical practice and research studies. It provides measures of vestibular-spinal function, giving complementary information that is indispensable for the assessment of patients with dizziness, in addition to analyzing sensorial interactions [9,15].

Stabilometry assesses posture balance through the quantification of posture oscillations from the orthostatic position in a force platform. It involves monitoring of pressure centers (CP) displacement to the lateral direction $(\mathrm{X})$ and anterior-posterior direction (Y). Normally, the tests are applied under different protocols for the support base (feet together, separated, supported by one foot, etc.), surface (hard or foam) and vision (opened and closed eyes) [11].

They were instructed, in case of use of labyrinthic depressing drug, to interrupt it for at least three days before the test and to avoid alcoholic drinks, non-essential drugs, coffee, tea or chocolate and smoking for 24 hours before the test.

Posturography was performed in a silent environment, with temperatures at about $25^{\circ} \mathrm{C}$. Before the conduction of the exams, the subjects remained seating down at rest for 5 minutes. During the exam, they were asked to take an orthostatic position over the platform, without shoes, feet separated at $30^{\circ}$ and heels together, relaxed arms along the body, a position that they should hold for about 1 minute. It was conducted both with eyes open and with eyes closed. The patients were allowed to step down from the force platform and relax for three minutes inbetween the tests (opened eyes, closed eyes).

The analyzed parameters were: 1 . Average amplitude of pressure center displacement at lateral plan (AMX). 2. Average amplitude of pressure center displacement at antero-posterior plan (AMY). 3. Average speed of pressure center displacement at lateral plan (VMX). 4. Average speed at pressure center displacement at anteroposterior plan (VMY). 5. Elliptical area of pressure center displacement at platform plan (EA). 
Table 1. Baseline Characteristics of the Study Population

\begin{tabular}{cccc}
\hline & The patients $(\mathrm{N}=40)$ & The controls $(\mathrm{N}=63)$ & P Value \\
\hline Age(years) & $68.33 \pm 10.00$ & $59.75 \pm 12.31$ & 0.140 \\
Gender(male/female) & $21 / 19$ & $25 / 38$ & 0.592 \\
Height(cm) & $160.88 \pm 10.61$ & $160.81 \pm 8.06$ & $0.022^{*}$ \\
& & With opened eyes & 0.062 \\
AMX(cm) & $3.71 \pm 1.31$ & $3.14 \pm 1.10$ & 0.054 \\
AMY(cm) & $3.68 \pm 1.49$ & $3.02 \pm 1.05$ & 0.124 \\
VMX(cm) & $1.42 \pm 0.58$ & $1.16 \pm 0.46$ & $0.010^{*}$ \\
VMY(cm) & $1.46 \pm 0.91$ & $1.06 \pm 0.49$ & 0.062 \\
EA(cm $\left.{ }^{2}\right)$ & $10.26 \pm 7.88$ & $7.25 \pm 5.70$ & 0.130 \\
AMX(cm) & $4.96 \pm 1.89$ & With closed eyes & $0.031^{*}$ \\
AMY(cm) & $4.75 \pm 1.91$ & $4.07 \pm 1.45$ & $0.008^{*}$ \\
VMX(cm) & $1.96 \pm 0.93$ & $4.01 \pm 1.30$ & $0.012^{*}$ \\
VMY(cm) & $2.08 \pm 1.05$ & $1.57 \pm 0.55$ & $1.52 \pm 0.67$ \\
EA(cm $\left.{ }^{2}\right)$ & $17.85 \pm 13.97$ & $12.55 \pm 9.71$ & 0.063 \\
\hline
\end{tabular}

Data presented mean \pm S.D (Standard deviation); AMX: Average amplitude of pressure center displacement at lateral plan, AMY: Average amplitude of pressure center displacement at antero-posterior plan, VMX: Average speed of pressure center displacement at lateral plan, VMY: Average speed at pressure center displacement at antero-posterior plan, EA: Elliptical area of pressure center displacement at platform plan; *P < 0.05

\subsection{Statistics}

Statistical analyses were carried out with the using the SPSS 18.0 (SPSS, Chicago, IL) package for Windows. Continuous variables were compared by ANOVA with Dunnett's posthoc analysis. Categorical variables were compared by $X^{2}$ testing. Statistical significance was set at $\mathrm{p}<0.05$.

\section{Results}

The prevalence of self-reported complaints suggestive for parkinsonism appears 38.84\% (40/103), even in persons whose total score of UPDRS is zero. There were 40 subjects that formed the patients with dizziness and subjective complaints related to typical clinical signs of PD (21 women and 19 men) aged on average 68.33 \pm 10.00 years. The control group comprised 63 subjects with dizziness and without such complaints (25 women and 38 men) aged on average $59.75 \pm 12.31$ years.

Baseline characteristics of the study population are shown in Table 1. It shows that the patients with dizziness and subjective complaints related to typical clinical signs of PD were significantly higher than the controls ( $p$ $=0.022)$ but age $(\mathrm{p}=0.140)$ and gender distribution $(\mathrm{p}=$ 0.592) were not significantly different.

We made a comparative analysis between the patients and the controls, comprising the five stabilometric parameters. Figure 1 and Figure $\mathbf{2}$ showed the parameter of average speed of pressure center displacement to the lateral plan (VMX) ( $\mathrm{p}=0.008$ with closed eyes) and antero-posterior plan (VMY) ( $\mathrm{p}=0.010$ with opened eyes, $\mathrm{p}=0.012$ with closed eyes), which presented statistically significant differences between the groups except VMX with opened eyes $(p=0.124)$.

And we analyzed the parameter of average amplitude of pressure center displacement to the lateral plan (AMX) and anteroposterior plan (AMY), and the elliptical pressure center displacement to the platform plan (EA) which presented compatible results with those in the previous parameters, but not statistically significant differences between the groups except AMY with closed eyes $(\mathrm{P}=0.031)$.

\section{Discussion}

We assessed stabilometric parameters of patients with dizziness and subjective complaints related to typical clinical signs of PD and compared them with the control group of patients with dizziness and without such complaints. In this study, 38.84\% of the participants experienced at least one of stiffness, slowness, tremors, or postural instability even without any parkinsonian signs on clinical examination. Our results showed that the patients had different results in some parameters, and they presented higher instability in the orthostatic position when compared to the control group.

It could support the notion that clinically manifest PD is preceded by a preclinical phase that is not entirely asymptomatic [1]. Subjective complaints related to typical clinical signs of PD might indicate a very early phase of not-yet-diagnosable PD, but may result in subtle signs that are very mild or only intermittently present and therefore not likely to be detected in routine screening or examination. It has been observed that prior to developing clinically manifest $\mathrm{PD}$, many patients experience a range of nonspecific symptoms, such as depression, fatigue, anxiety or pain including dizziness [6,7]. Whitney [8] mentioned dizziness as one of the most frequent complaints in his study.

Dizziness may be a very early symptom that progresses to overt postural instability and an increased risk 


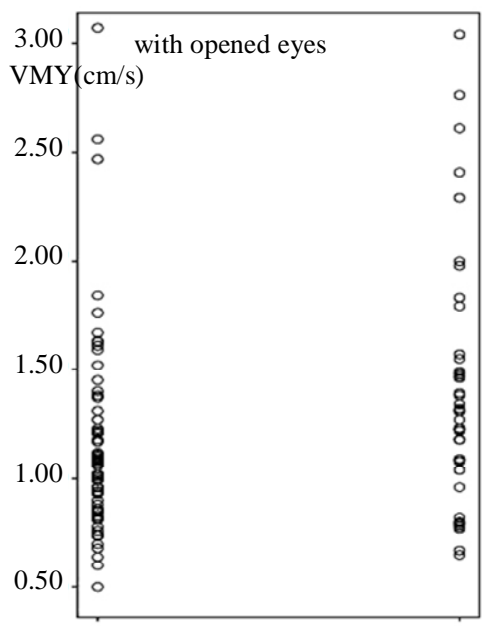

(a)

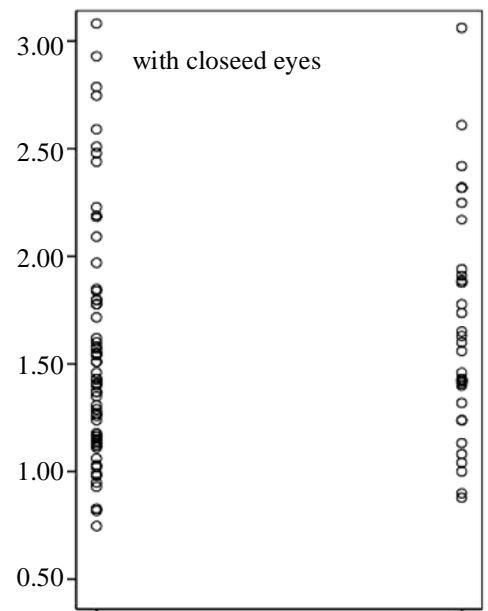

(a) (b)

Figure 1. Comparison of mean speed of pressure center displacement at lateral plan (VMX) between the patients (b) and cont rols $(a)$ with opened eyes $(p=0.124)$ and closed eyes $(p=0.008)$.

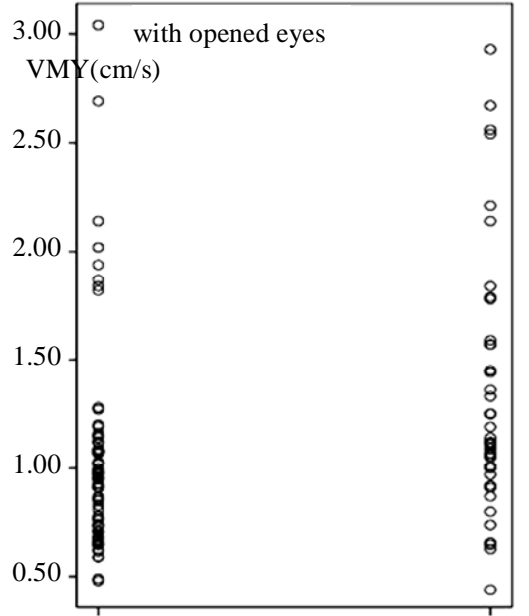

(a)

(b)

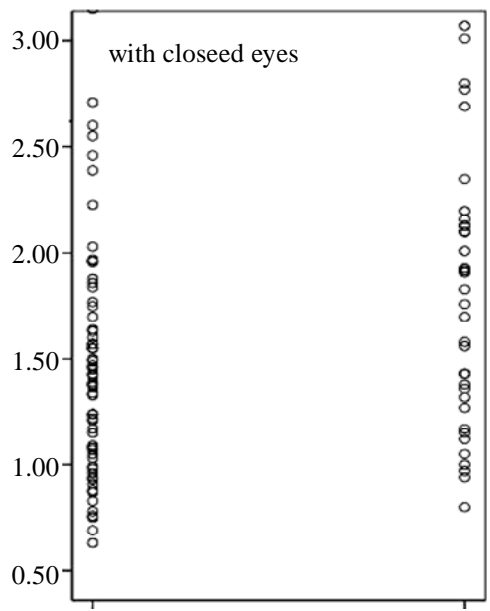

(a)

(b)

Figure 2. Comparison of mean speed of pressure center displacement at antero-posterior plan (VMY) between the patients (b) and controls $(a)$ with opened eyes $(p=0.010)$ and closed eyes $(p=0.012)$.

of falling later. Postural stability depends on the integrity of the vestibular system (vestibular labyrinth, vestibulocochlear nerve, nuclei, channels and interrelations in the central nervous system), the somatosensory system (sensory receptors located in the tendons, muscles and articulations) and vision [16]. Symptoms like dizziness can occur as a consequence of such alterations in those systems. In PD, there could be a preclinical vulnerability in the ability of the central nervous system to process vestibular, visual and proprioceptive signs which are responsible for the maintenance of balance, with a decrease in the capacity to modify the postural adaptive reflexes.

Some prospective studies have been observed that Alzheimer disease or cerebral small vessel disease that showed that subjective memory complaints in persons without objective cognitive impairment were associated with an increased risk of developing dementia or more white matter lesions [17-20]. Both PD and Alzheimer disease are characterized by a phase of neuronal degeneration and loss of function before the appearance of typical symptoms [21], and apparently, such a preclinical phase also exists in slowly progressive the neurodegeneraive disease. The neurodegeneration could manifest itself by a decline in the functions of several organs in a linear fashion in function of the time with the exact transition point not definable.

In our study, we hypothesized that mild dopamine deficiency in preclinical PD might result in subtle subjec- 
tive complaints. However it must be supported further studies using several potential biomarkers for preclinical PD such as loss of the dopamine transporter detected by positron emission tomographic imaging, subtle abnormalities on psychological testing, olfactory dysfunction, and biochemical markers in serum or cerebrospinal fluid [22,23].

Another aspect that we took into account during the assessment was the effect of visual deprivation. In our statistical analysis, we observed that the patients had statistically significant results for the parameters of average speed of pressure center displacement to the anteroposterior plan (VMY), to the lateral plan (VMX) and the parameter of average amplitude of pressure center displacement to the antero-posterior plan (AMY) presented mean values with closed eyes compared to with opened eyes. However, only VMY was higher significantly for opened eyes than for closed eyes. The absence of statistically significant results for other parameters may be justified by the small sample size. It is expected that subjects with subjective complaints related to typical clinical signs of PD have more visual dependency and therefore, upon closing their eyes, they present more affections in their stabilometric parameters [24].

We have a lot of limitiations. First of all, it needs the follow-up study to exam whether dizziness as subtle subjective complaint in preclinical PD is associated with an increased risk of PD in the future. Unfortunately, we could not specify the types of dizziness and the subjective complaints. Dizziness is a subjective and nonspecific symptom with varied characteristics. It may be described as a sensation of imbalance, instability, fluctuation, rotation, "empty head", among others. We assessed dizziness in a stepwise fashion by asking participants to specify, as mentioned in methods, but didn't analysis separately. And we could not use symptom-specific questions to access of subjective complaints related to typical clinical signs of PD to them. We continue to follow up participants after, which may enable us to support or revise the study on the base of additional information if necessary.

In this study, the patients with dizziness and subjective complaints related to typical clinical signs of PD complaints presented higher instability in the orthostatic position than the control group of patients with dizziness and without such complaint. It could suggest that dizziness may be one symptom of preclinical PD and progress to overt postural instability. It is believed that a vestibular approach in preclinical PD is required.

\section{Acknowledgements}

This study was supported by a grant of the Korea Health 21 R\&D Project, Ministry of Health \& Welfare, Republic of Korea (A050079).

\section{REFERENCES}

[1] S.T. DeKosky, K. Marek, "Looking Backward to Move Forward: Early Detection of Neurodegenerative Disorders”, Science, Vol. 302, No.5646, 2003, pp. 830-834. doi:10.1126/science.1090349

[2] E. C. Wolters, C. Francot, P. Bergmans, A. Winogrodzka, J. Booij, H. W. Berendse, H. W. Berendse, J. C. Stoof, "Preclinical (premotor) Parkinson's Disease," Journal of Neurology, Vol. 247, No. 2, 2000, pp. 103-109.

[3] M. M. Ponsen, D. Stoffers, J. Booij, B. L. van Eck-Smit, E. Wolters and H. W. Berendse, "Idiopathic Hyposmia as a Preclinical Sign of Parkinson's Disease,” Annals of Neurology, Vol. 56, No. 2, 2004, pp. 173-181. doi:10.1002/ana.20160

[4] G. Glosser, C. Clark, B. Freundlich, L. Kliner-Krenzel, P. Flaherty and M. Stern, "A Controlled Investigation of Current and Premorbid Personality: Characteristics of Parkinson's Disease Patients”, Movement Disorders, Vol. 10, No. 2, 1995, pp. 201-206.

[5] G. W. Paulson and N. Dadmehr, "Is there a Premorbid Personality Typical for Parkinson's Disease?” Neurology, Vol. 41, No. 5, 1991, pp. 73-76.

[6] A. F. Leentjens, M. Van den Akker, J. F. Metsemakers, R. Lousberg and F. R. Verhey, "Higher Incidence of Depression Preceding the Onset of Parkinson's Disease: A Register Study,” Movement Disorders, Vol. 18, No. 4, 2003, pp. 414-418. doi:10.1002/mds.10387

[7] E. G. Gonera, M. van Hof, H. J. Berger, C. van Weel an d M. W. Horstink, "Symptoms and Duration of the Prodromal Phase in Parkinson's Disease," Movement Disorders, Vol. 12, No.6, 1997, pp. 871-876. doi:10.1002/mds.870120607

[8] S.L. Whitney. "Treatment of Elderly Patients with Vestibular Dysfunction”. In: S.J. Herdman, $2^{\text {nd }}$ ed., Vestibular Rehabilitation, São Paulo: Manole, 2002, pp. 505-528.

[9] P-M. Gagey. "Non-vestibular Dizziness and Static Posturography”, Acta Oto-Rhino-Laryngologica Belgica, Vol. 45, 1991, 335-339.

[10] N. C. Zhong and J. Jin. "New Posture Equilibrometer for the Diagnosis of Vestibular Disease,” European Archives of Oto-Rhino-Laryngology, Vol. 248, No. 3, 1991, pp. 135-138. doi:10.1007/BF00178922

[11] R. M. Kantner, A. M. Rubin, C. W. Armstrong and V. Cummings, "Stabilometry in Balance Assessment of Dizzy and Normal Subjects," American Journal of Otolaryngology, Vol. 12, No. 4, 1991, pp. 196-204. doi:10.1016/0196-0709(91)90120-5

[12] J.G. Douglas, O. Eugene, G. Sid. "Diagnostic Criteria for Parkinson Disease”, Archives of Neurology, Vol. 56, No. 1, 1999, pp. 33-39. doi:10.1001/archneur.56.1.33

[13] S. Fahn and R. L. Elton, "UPDRS Development Committee. Unified Parkinson's DiseaseRating Scale,” In: S. Fahn, C.D. Marsden and D.B. Calne, Eds., Recent Developments in Parkinson's Disease, Vol. 2, London, Eng- 
land: MacMillan, 1987, pp. 153-163.

[14] B. H. Wood, J. A. Bilclough, A. Bowron and R. W. Walker, "Incidence and Prediction of Falls in Parkinson's Disease: A Prospective Multidisciplinary Study,” Journal of Neurology, Neurosurgery, and Psychiatry, Vol. 72, No. 6, 2002, pp. 721-725. doi:10.1136/jnnp.72.6.721

[15] M. E. Norré and G. Forrez, "Posture Testing (Posturography) in the Diagnosis of Peripheral Vestibular Pathology,” Archives of Otolaryngology, Vol. 243, No. 3, 1986, pp. 186-189.

[16] F. F. Ganança, A. S. O. Castro, F. C. Branco and J. Natour, "Interference from the Patient's Quality of Life of Patients with Chronic Peripheral Vestibular Area," Revista Brasileira de Oto-Rino-Laringologia, Vol. 70, No.1, 2004, pp. 94-101.

[17] M. I. Geerlings, C. Jonker, L. M. Bouter, H. J. Ader an d B. Schmand, "Association between Memory Complaints and Incident Alzheimer's Disease in Elderly People with Normal Baseline Cognition," The American Journal of Psychiatry, Vol. 156, No. 4, 1999, pp. 531537.

[18] A. F. Jorm, K. H. Masaki, D. G. Davis, J. Hardman, J. Nelson, W. R. Markesbery, H. Petrovitch, G. W. Ross an d L. R. White, "Memory Complaints in Nondemented Men Predict Future Pathologic Diagnosis of Alzheimer Disease," Neurology, Vol. 63, No.10, 2004, pp. 19601961.

[19] C. Jonker, M. I. Geerlings and B. Schmand, “Are Mem- ory Complaints Predictive for Dementia? A Review of Clinical and Population-Based Studies," International Journal of Geriatric Psychiatry, Vol. 15, No. 1, 2000, pp. 983-991.

doi:10.1002/1099-1166(200011)15:11<983::AIDGPS238>3.0.CO;2-5

[20] J. C. de Groot, F. E. de Leeuw, M. Oudkerk, A. Hofman, J. Jolles and M. M. Breteler, "Cerebral White Matter Lesions and Subjective Cognitive Dysfunction: The Rotterdam Scan Study,” Neurology, Vol. 56, No.11, 2001, pp. 1539-1545.

[21] S. T. DeKosky and K. Marek, "Looking Backward to Move Forward: Early Detection of Neurodegenerative Disorders,” Science, Vol. 302, No. 5646, 2003, pp. 830 834. doi:10.1126/science.1090349

[22] M. M. Ponsen, D. Stoffers, J. Booij, B. L. van Eck-Smit, E. Wolters and H. W. Berendse, "Idiopathic Hyposmia as a Preclinical Sign of Parkinson's Disease," Annals of Neurology, Vol. 56, No.2, 2004, pp. 173-181. doi:10.1002/ana.20160

[23] A. W. Michell, S. J. Lewis, T. Foltynie and R. A. Barker. "Biomarkers and Parkinson's Disease," Brain, Vol. 127, No. 8, 2004, pp. 1693-1705.

[24] M. A. Sanz, R. B. Guzman, C. C. Cerverón and J. M. Baydal, "Analysis of the Visuo-Vestibular Interaction and Visual Influence Postural Control,” Acta Otorrinolaringológica Española, Vol. 55, 2004, pp. 9-16. 